\title{
Non-Small Cell Lung Carcinoma Presenting With Severe Tracheal Deviation: A Case Report
}

\author{
Oluwatoyin Dada ${ }^{1}$, Steven Douedi ${ }^{1}$, Tiffany Purewal ${ }^{1}$, Mohammad Hossain ${ }^{2}$ \\ 1. Internal Medicine, Hackensack Meridian Health, Jersey Shore University Medical Center, Neptune, USA 2. Internal \\ Medicine, Jersey Shore University Medical Center, Neptune, USA
}

Corresponding author: Oluwatoyin Dada, toy.d993@gmail.com

\begin{abstract}
Non-small cell lung carcinoma (NSCLC) is usually diagnosed in adults between the ages of 18-35 years. Here, we present a case of a young adult who presented with severe tracheal deviation and was diagnosed with advanced-stage NSCLC with no past medical or social histories; she presented with worsening right arm pain, numbness, and tingling over the course of three weeks. She presented to an urgent care center that prescribed her nonsteroidal anti-inflammatory drugs (NSAIDs) and muscle relaxants without any symptom improvement. Her pain continued to worsen, and she went to the emergency department (ED) where an X-ray and CT angiogram were performed revealing a large right lung mass severely compressing the trachea. Biopsies confirmed non-small cell lung cancer and the patient was started on chemotherapy. As clinicians, sometimes we do not pay attention to young patients with nonspecific complaints. This case clearly reflects the importance of proper evaluation of symptoms even though there is low clinical suspicion.
\end{abstract}

Categories: Internal Medicine, Radiology, Oncology

Keywords: non-small cell lung carcinoma, tracheal deviation, lung cancer, adenocarcinoma, malignancy

\section{Introduction}

Non-small cell lung carcinoma (NSCLC) makes up about $80 \%$ of all lung cancer diagnoses. NSCLC is divided into multiple subtypes, with adenocarcinomas being the most diagnosed. Features of adenocarcinomas include, but are not limited to peripheral masses, subpleural nodules, or malignant pleural effusions. In terms of demographics, females are more affected compared to males. It has been noted that women with adenocarcinoma are often non-smokers, suggesting that other factors are at play, including mutations or hormonal differences [1].

We present a case of a young African-American woman who initially presented with pain and numbness of her right arm without respiratory symptoms and found to have severe tracheal deviation due to stage IV lung adenocarcinoma.

Received 04/16/2020

\section{Case Presentation}

A 24-year-old Haitian female with no known past medical history or smoking history was in her usual state of health until three weeks prior to presentation when she started to experience right arm pain, numbness, and tingling sensation. She did not report a history of cancer in her family. She initially presented to an urgent care facility where she was prescribed nonsteroidal anti-inflammatory drugs (NSAIDs) and muscle relaxants without any improvement of her symptoms. She also had two episodes of pre-syncope over the past two months which she attributed to poor diet; she did not seek any medical attention. On the day of presentation to the emergency department (ED), the patient was returning from the Bahamas via airplane when she felt progressively worsening right-sided chest discomfort radiating to the right flank and back. She reported right upper extremity pain and numbness. She did not report cough, fever, shortness of breath, cold symptoms or recent changes in the weight and appetite. Her vitals in ED were a heart rate of 120 beats per minute, oxygen saturation of $98 \%$ on room air, blood pressure of $110 / 78 \mathrm{~mm} \mathrm{Hg}$, and temperature of 98.2 F. Her physical examination was unremarkable. Initial laboratory investigation was normal including complete blood count (CBC) and comprehensive metabolic panel (CMP). A preliminary chest X-ray was done (Figure 1). 


\section{Cureus}

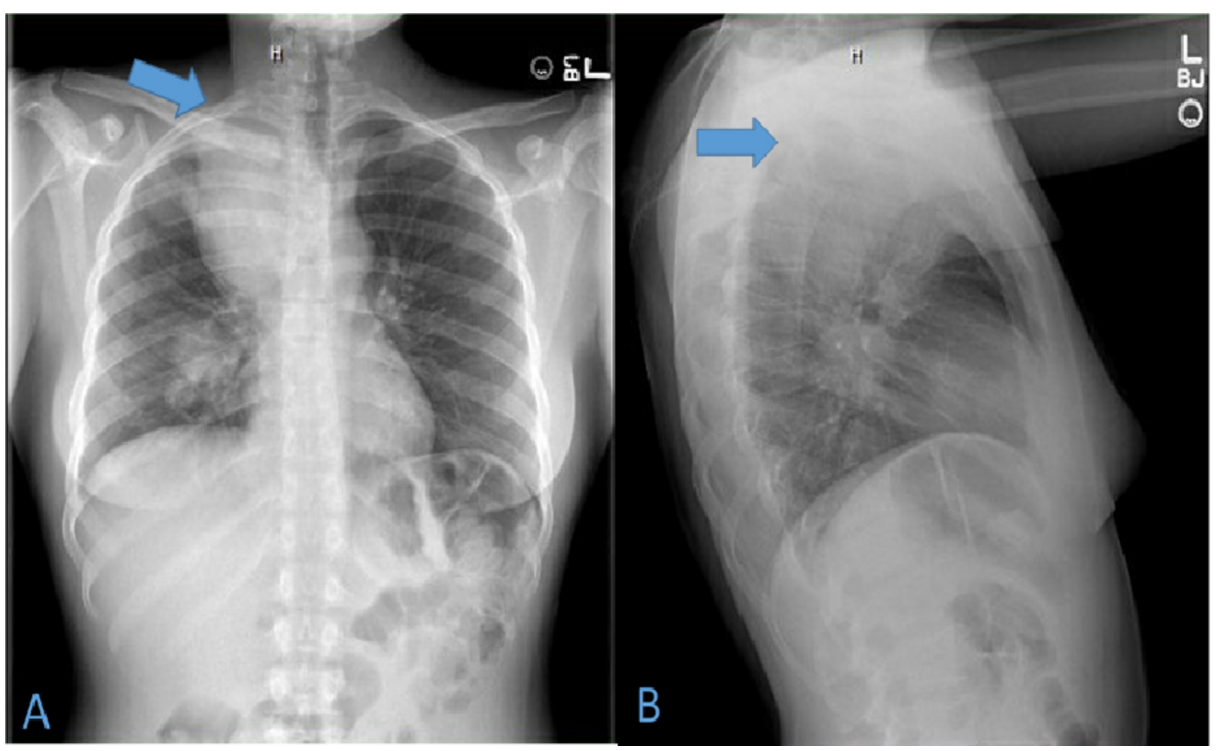

\section{FIGURE 1: Postero-anterior and lateral view of chest X-ray}

1A. Postero-anterior view of the chest showing the right upper lung mass, indicated by the blue arrow. 1B. Lateral view of the chest showing the right upper lung mass, indicated by the blue arrow.

Due to tachycardia and positive travel history, a computed tomography angiogram (CTA) was performed which was negative for a pulmonary embolism. However, CTA revealed a heterogeneous superior right mediastinal mass, measuring about $10 \mathrm{~cm}$. The trachea was noted to be severely deviated to the left and markedly stenosed. Multiple pleural-based lung masses and nodules were also present on imaging, which are classical imaging signs of adenocarcinoma (Figure 2).

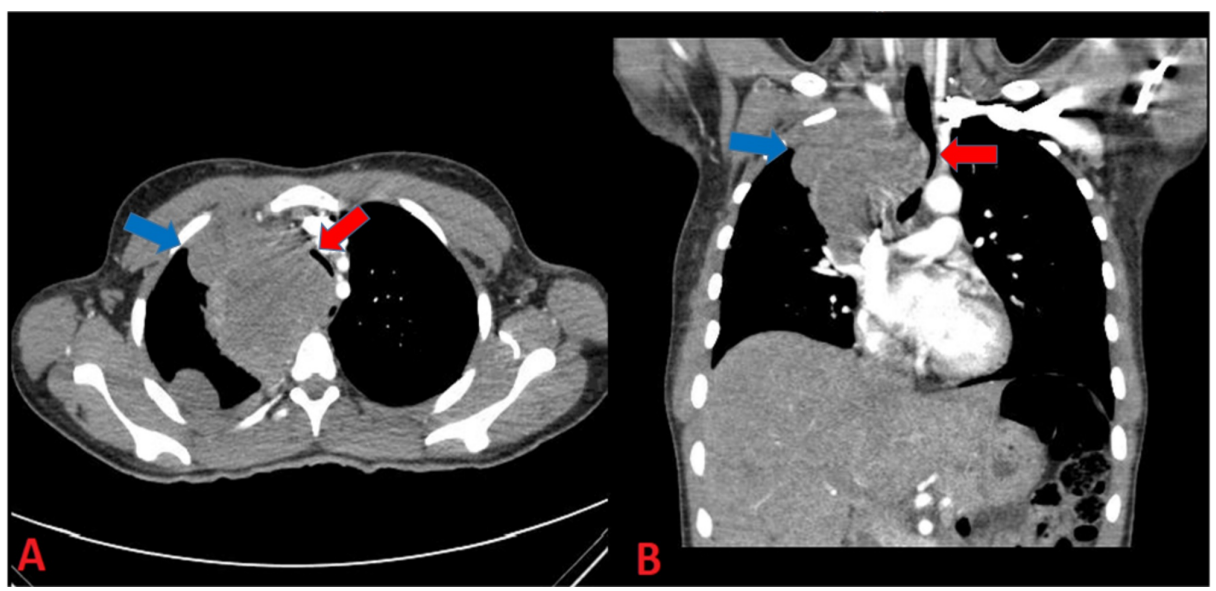

\section{FIGURE 2: Computed tomography angiography (CTA) of the chest (axial} and sagittal view)

1A. CT scan of chest showing a heterogeneous superior right mediastinal mass measuring $10 \mathrm{~cm}$ craniocaudally and a $3.6 \mathrm{~cm}$ mass in the right middle lobe (blue arrow) and severe tracheal stenosis (red arrow). 1B. CT scan of chest showing lung mass (blue arrow) compression leading to "coin slit" trachea (red arrow).

The patient underwent a mediastinal biopsy and was diagnosed with metastatic poorly differentiated nonsmall cell adenocarcinoma (Figure 3). She later underwent a bronchoscopy with tracheal stent placement. Her right arm pain, numbness, and tingling eventually resolved after treatment with systemic steroids. She is now undergoing systemic chemoimmunotherapy with carboplatin/pemetrexed although she has recurrent admissions to the hospital for chronic malignant pleural effusions and chest pain, requiring tunneled pleural catheter placement. 


\section{Cureus}

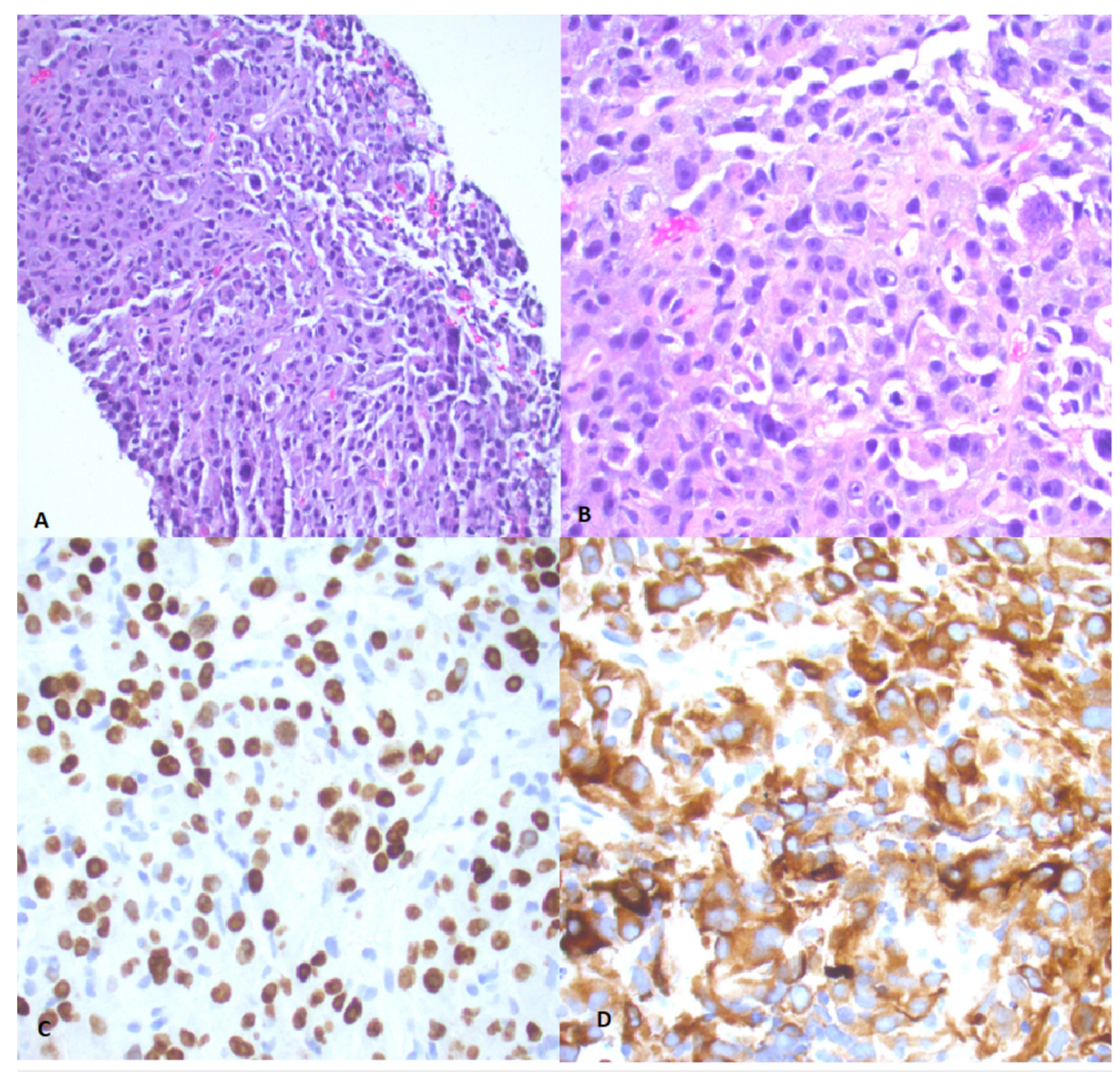

\section{FIGURE 3: Mediastinal biopsy tissue}

A: Gross tissue sample demonstrating poorly-differentiated non-small cell lung carcinoma (NSCLC); B: This is a close-up of the cellular composition showing pleomorphic, hyperchromatic nuclei, variably prominent nucleoli, and small to moderate amounts of cytoplasm. These are all classic hallmarks of NSCLC; C: Positive TTF-1 staining; D: Positive cyto-keratin AE1/AE3

\section{Discussion}

Literature about the clinical characteristics, pathophysiology, and time trends of young patients with lung cancer is very limited, having only a 1.37\% incidence rate in ages of 18 to 35 [2]. However, one study called the Surveillance, Epidemiology and End Results (SEER) database created in 2010 gives us some insight regarding the diagnosis of NSCLC in young adults. Younger patients were more likely to have adenocarcinoma, while older patients were more likely to have squamous cell carcinoma [3]. Generally, about $70 \%$ of patients diagnosed with lung cancer present with advanced disease [4]. More specifically, younger patients make up about $0.6 \%$ of NSCLC diagnoses and were more likely to present with advancedstage NSCLC. When race was taken into account, female African-Americans and Pacific Islanders were the most likely to present with advanced features, similar to our patient [3].

One study, looking at features of NSCLC in young patients, noted that non-smokers were less likely to have upper lobe cancers, which our patient presented with on imaging [5]. It has been hypothesized that nonsmokers who have lung cancer have mechanisms of disease that are different from patients with a smoking history. Researchers have proposed risk factors for the development of NSCLC in never-smokers are weak to modest at best and require more investigation [6]. One suggested mechanism of carcinogenesis is the upregulation of estrogen-alpha and -beta receptors in cancer cells, which may explain why the incidence of NSCLC is higher in non-smoking women [7]. Another mechanism to consider is the epidermal growth factor receptor (EGFR) mutation pathway that appears to be affected in non-smoking patients who develop adenocarcinoma [3]. Another risk factor that has been suggested is that women are likely to be more exposed to cooking fumes, which may be associated with a higher risk of lung cancer [6].

NSCLC is commonly treated with surgery and adjuvant therapy from stages I-IIIA. For stage IV patients, like our patient, the first-line therapy is usually platinum plus pemetrexed, especially if a patient has confirmed adenocarcinoma. Though this is a recommended first-line treatment, other chemotherapeutics were found to be equally efficacious. The median lifespan after starting treatment is eight to ten months [7]. Other studies have shown statistical significance with treatment of lung cancer with platinum-based regimens 
Our young African-American female patient, presented in this case report, had no modifiable risk factors to develop NSCLC. On presentation, her only symptom was right arm pain without any respiratory complaints or symptoms. Despite this benign appearing presentation, she was found to have severe tracheal stenosis due to a large lung mass, which do not commonly present together in lung cancer. One case report presents a case of a gentleman who had right shoulder pain and was found to have lung cancer that presented as metastatic disease to the scapula and spine. He did not have any tracheal deviation as a result [9]. Another case report presents a case in which a woman desaturated during bronchoscopy secondary to tracheal stenosis secondary to lung cancer; however, she did not report any pain symptoms [10]. In terms of prognosis, young non-smokers with regional disease had better survival outcomes [3].

\section{Conclusions}

The low prevalence of non-small cell lung cancer in the young patient population combined with the differences in clinical presentation described in this case makes this a very imperative topic for the healthcare team to be aware of to prevent delayed diagnosis and treatment as well as to improve patient outcomes. Additionally, a better understanding of other risk factors, besides age, smoking, exposures to chemicals, etc., is needed in order to develop targeted therapeutics.

\section{Additional Information \\ Disclosures}

Human subjects: Consent was obtained by all participants in this study. Conflicts of interest: In compliance with the ICMJE uniform disclosure form, all authors declare the following: Payment/services info: All authors have declared that no financial support was received from any organization for the submitted work. Financial relationships: All authors have declared that they have no financial relationships at present or within the previous three years with any organizations that might have an interest in the submitted work. Other relationships: All authors have declared that there are no other relationships or activities that could appear to have influenced the submitted work.

\section{References}

1. Torre LA, Siegel RL, Jemal A: Lung cancer statistics. Lung Cancer and Personalized Medicine. Ahmad A, Gadgeel S (ed): Springer, Cham; 2016. 893:1-19. 10.1007/978-3-319-24223-1_1

2. Liu B, Quan X, Xu C, Lv J, Li C, Dong L, Liu M: Lung cancer in young adults aged 35 years or younger: a fullscale analysis and review. J Cancer. 2019, 10:3553-3559. 10.7150/jca.27490

3. Subramanian J, Morgensztern D, Goodgame B, Baggstrom MQ, Gao F, Piccirillo J, Govindan R: Distinctive characteristics of non-small cell lung cancer (NSCLC) in the young: a Surveillance, Epidemiology, and End Results (SEER) analysis. J Thorac Oncol. 2010, 5:23-28. 10.1097/JTO.0b013e3181c41e8d

4. Molina JR, Yang P, Cassivi SD, Schild SE, Adjei AA: Non-small cell lung cancer: epidemiology, risk factors, treatment, and survivorship. Mayo Clin Proc. 2008, 83:584-94. 10.4065/83.5.584

5. Thomas A, Chen Y, Yu T, Jakopovic M, Giaccone G: Trends and characteristics of young non-small cell lung cancer patients in the United States. Front Oncol. 2015, 5:113. 10.3389/fonc.2015.00113

6. Subramanian J, Govindan R: Lung cancer in never smokers: a review . J Clin Oncol. 2007, 25:561-570. 10.1200/JCO.2006.06.8015

7. Zappa C, Mousa SA: Non-small cell lung cancer: current treatment and future advances . Transl Lung Cancer Res. 2016, 5:288-300. 10.21037/tlcr.2016.06.07

8. Wheatley-Price P, Blackhall F, Lee SM, et al.: The influence of sex and histology on outcomes in non-smallcell lung cancer: a pooled analysis of five randomized trials. Ann Oncol. 2010, 21:2023-2028. 10.1093/annonc/mdq067

9. Demetrious J, Demetrious GJ: Lung cancer metastasis to the scapula and spine: a case report . Chiropr Osteopat. 2008, 16:8. 10.1186/1746-1340-16-8

10. Himeji D, Tanaka G, Fukuyama C, Yamanaka A, Shiiba R, Moriguchi S, Marutsuka K: Severe tracheal stenosis after first administration of pembrolizumab rescued by Dumon Y-stent in a lung cancer patient. Respir Med Case Rep. 2019, 28:100923. 10.1016/j.rmcr.2019.100923 